\title{
Erratum
}

\section{Modellrechnungen zur symmetrischen Vierzentrenbindung. II}

\author{
P. KADURA
}

Theoret. chim. Acta (Berl.) 8, 292 (1967)

Fingegangen am 10. November 1967

Die Konfiguration $\Phi_{2}(1,2)$ besitzt nicht die Symmetrie $A_{1}$. Die richtige Konfiguration muß heißen:

$$
\Phi_{2}(1,2)=\frac{1}{\sqrt{3}}\left[\psi_{2}(1) \psi_{2}(2)+\psi_{3}(1) \psi_{3}(2)+\psi_{4}(1) \psi_{4}(2)\right]
$$

Dadurch ergeben sich geringfügige Änderungen in den numerischen Ergebnissen. Für $H_{2 a}$ und $H_{12}$ erhält man jetzt:

$$
\begin{aligned}
H_{22} & =\frac{2}{1-S}\left[J_{1}-J_{2}+J_{3}-2 J_{4}+3 J_{5}-2 J_{6}\right] \\
& +\frac{1}{(1-S)^{2}}\left[\frac{3}{4} J_{7}+\frac{1}{4} J_{8}-3 J_{9}+\frac{5}{2} J_{10}+J_{11}-2 J_{12}+\frac{1}{2} J_{13}\right], \\
H_{12} & =\frac{\sqrt{3}}{(1+3 S)(1-\bar{S})}\left[\frac{1}{4} J_{7}-\frac{1}{4} J_{8}+J_{9}+\frac{1}{2} J_{10}-J_{11}-\frac{1}{2} J_{13}\right] .
\end{aligned}
$$

Die Grenzwerte für $R \rightarrow \infty$ lauten hierfür:

$$
\begin{aligned}
\lim _{R \rightarrow \infty} H_{22} & =-1+\frac{15}{32} & \lim _{R \rightarrow \infty} H_{12} & =\sqrt{3} \cdot \frac{5}{32} \\
\lim _{R \rightarrow \infty} E_{1_{A_{1}}, 1} & =-1 & \lim _{R \rightarrow \infty} E_{1} A_{A_{1}, 2} & =-1+\frac{5}{8} .
\end{aligned}
$$

Die Fig. 2 und 3 bleiben riohtig, da die Änderungen nahezu unter der Zeichengenauigkeit liegen. Eine Übersicht über die Änderungen der entscheidenden Größen gibt folgende Tabelle:

\begin{tabular}{lllllll}
\hline & $R_{\min }$ & $\alpha$ & $f\left(R_{\min }\right)$ & $R_{\max }$ & $\alpha$ & $f\left(R_{\max }\right)$ \\
\hline alt & $2,47 \mathrm{AE}$ & 1,28 & $0,0348 \mathrm{AE}$ & $6,09 \mathrm{AE}$ & 1 & $0,1291 \mathrm{AE}$ \\
neu & $2,47 \mathrm{AE}$ & 1,28 & $0,0331 \mathrm{AE}$ & $6,11 \mathrm{AE}$ & 1 & $0,1284 \mathrm{AE}$
\end{tabular}

\begin{tabular}{lll}
\hline & $D_{\mathrm{e}}$ & $R_{\mathrm{k} \mathrm{kt}}$ \\
\hline alt & $0,0943 \mathrm{AE}=2,56 \mathrm{eV}$ & $0,1291 \mathrm{AE}=3,51 \mathrm{eV}$ \\
neu & $0,0953 \mathrm{AE}=2,59 \mathrm{eV}$ & $0,1284 \mathrm{AE}=3,49 \mathrm{eV}$
\end{tabular}

